\title{
A higher spirit: avoiding yeast suicide during alcoholic fermentation
}

\author{
D Carmona-Gutierrez ${ }^{1}$, C Sommer ${ }^{1}$, A Andryushkova ${ }^{1}$, G Kroemer ${ }^{2,3,4,5,6}$ and F Madeo ${ }^{\star, 1}$ \\ Cell Death and Differentiation (2012) 19, 913-914; doi:10.1038/cdd.2012.31
}

Alcoholic fermentation probably represents the oldest biotechnological utilization of a microorganism, even beyond history recording. Over time, yeast - especially the budding yeast Saccharomyces cerevisiae - has gained a unique role of cultural, social and industrial importance that ranges from its use in the millenary art of brewing beer and making wine to its modern application in the production of ethanol-based biofuel. Massive production of ethanol as an eco-friendly liquid fuel might help counteract the alarming repercussions of climate change, as well as the persisting insecurity of petroleum markets, yet relies on increasing fermentation performance to maintain its economic feasibility. The same applies to the alcoholic beverage industry, where stuck or sluggish fermentation accounts for substantial economic losses. For this reason, resolving a basic yeast cell biology problem like fermentative limitation may have a major impact on the biotechnology industry. This limitation is mainly caused by stress situations during the fermentative process, such as high osmotic pressure, large $\mathrm{pH}$ changes or accumulating fermentation products that can compromise yeast cell growth and survival. Thus, improving tolerance of yeast cells to different stresses can be expected to amend fermentative efficiency.

Ethanol toxicity is one of the major stress factors that undermine the fermentative capability of $S$. cerevisiae. Although yeast cells dispose of a complex, though not fully understood set of ethanol stress responses, ${ }^{1}$ increasing ethanol concentrations resulting from fermentation eventually lead to growth inhibition and cell death, limiting the viable titer and the fermentative output. Hence, the identification and improvement of ethanol-specific stress responses represents a logical approach to pursue. However, when these adaptive responses fail, the ultimate consequence of ethanol toxicity is cell death. Thus, a complementary strategy that is yet to be explored would target the demise of $S$. cerevisiae cells, taking into account that yeast cell death is an active, molecularly controlled process and hence can be inhibited by pharmacological agents or genetic manipulations. ${ }^{2,3}$

In fact, ethanol-induced yeast cell death follows a regulated pathway that is characterized by morphological and biochemical markers of apoptosis (including mitochondrial fragmentation) and by a causal role of reactive oxygen species (ROS), ${ }^{4}$ which represent major regulators in the mitochondrial pathway of yeast apoptosis. ${ }^{5}$ Interestingly, a recent study using different enological strains of $S$. cerevisiae indicates that ROS production is a characteristic feature during hypoxic fermentation in highsugar-containing medium, which is in line with other studies indicating ROS production to occur in fermenting yeast. ${ }^{6}$ Given that yeast requires mitochondria for basic metabolism even under anaerobic conditions ${ }^{4,7}$ throughout the fermentation process, ${ }^{8}$ these data suggest that one possible target for inhibiting ethanol-induced cell death might be the mitochondrion. Intriguingly, different other stresses that usually prejudice industrial ethanol productivity, like hyperosmotic stress due to high initial substrate concentration, competitive interaction between yeast species, acetic acid stress or prolonged culture in stationary phase have been shown to result in PCD in $S$. cerevisiae, and in all cases, mitochondria seem to be implicated in the corresponding process. ${ }^{2}$

Indeed, mitochondria have a major role in stress-induced apoptosis and necrosis through damage inflicted upon ROS production, as well as via release of potentially harmful proteins upon permeabilization of mitochondrial membranes. ${ }^{2,5}$ As a result, mitochondrial membranes constitute a battleground on which multiple death-inducing and death-inhibitory factors determine a life/death decision at the molecular level. Consequently, the selective degradation of mitochondria (mitophagy), a process in which damaged or leaking mitochondria are sequestered by double-membrane compartments and then degraded by vacuolar/lysosomal enzymes, is an important stress response mechanism conserved from yeast to mammals. ${ }^{9,10}$ Thus, stimulating or inhibiting mitophagy or general autophagy (cellular self-digestion) can dramatically influence the propensity of cells to die.

In principle, two non-exclusive strategies may be employed to protect fermenting yeast from PCD: exogenous and endogenous intervention. First, a set of additives may be used to supplement cultures, for instance with the scope of scavenging ROS and thus neutralizing one of the major common stimulators of $\mathrm{PCD}$, or that of inducing the

\footnotetext{
${ }^{1}$ Institute of Molecular Biosciences, University of Graz, Graz, Austria; ${ }^{2}$ INSERM, U848, Villejuif, France; ${ }^{3}$ Metabolomics Platform, Institut Gustave Roussy, Villejuif, France; ${ }^{4}$ Centre de Recherche des Cordeliers, Paris, France; ${ }^{5}$ Pôle de Biologie, Hôpital Européen Georges Pompidou, AP-HP, Paris, France and ${ }^{6}$ Université Paris Descartes, Sorbonne Paris Cité, Paris, France

${ }^{*}$ Corresponding author: F Madeo, Institute of Molecular Biosciences (IMB), Karl-Franzens University, Humboldtstrasse 50/EG, Graz 8010, Austria. Tel: + 43316380 8878; Fax: + 43316380 9898; E-mail: frank.madeo@ uni-graz.at
} 


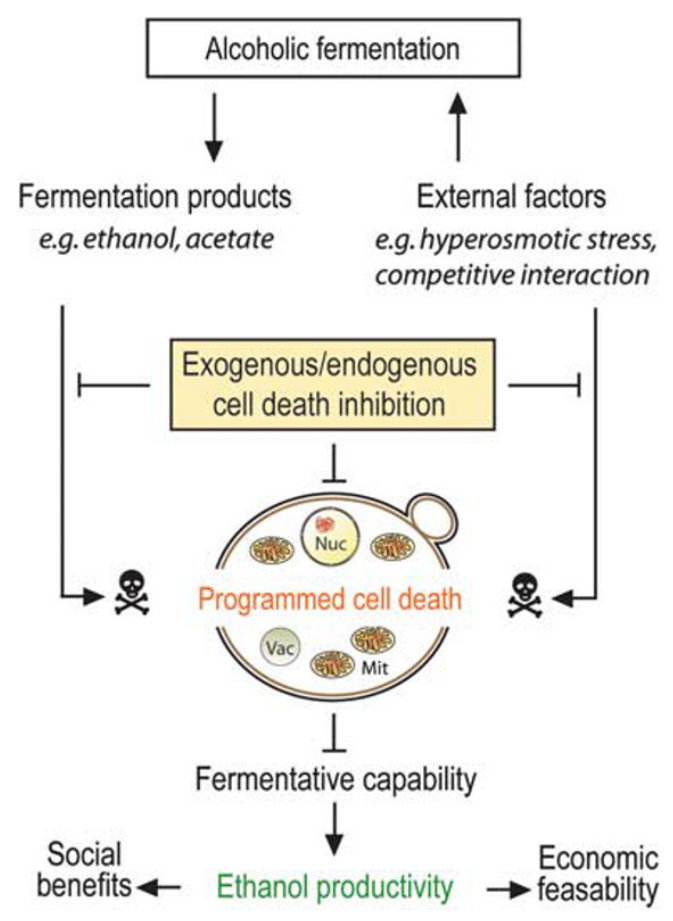

Figure 1 Yeast cell death and alcoholic fermentation. Fermentation products like acetate or ethanol and external factors such as hyperosmotic stress due to high initial substrate concentration or competitive interaction between yeast species may compromise fermentative capability. These factors can also induce yeast programmed cell death, meaning that pharmacological or genetic inhibition of this process might improve fermentation yields and thus increase ethanol production, resulting in the corresponding socio-economic benefits. Nuc: nucleus; Vac: vacuole; Mit: mitochondria

autophagic removal of damaged mitochondria. Second, genetic engineering may lead to the generation of yeast strains that are intrinsically resistant to PCD. This can be achieved by the transgenic overexpression of anti-apoptotic or anti-necrotic factors, including such that protect mitochondria against fragmentation/permeabilization. Alternatively, yeast cells can be protected against death by inactivating genes that code for proteins with potential lethal functions (cell death executors). Such lethal factors include proteins mediating mitochondrial fragmentation/permeabilization, as well as lethal proteases, nucleases and their co-activators. ${ }^{2}$

Summing up, the control of yeast population dynamics, which is crucial for the profitability of fermentation-based ethanol production, requires taking account of the contribution and relevance of yeast PCD in general and the lethal role of mitochondria in particular (Figure 1). Accordingly, genetic or pharmacological manipulations designed to suppress yeast PCD might contribute to attain higher fermentative yields. Thus, in this concrete case, it is avoiding death that may help achieve a higher spirit.

Acknowledgements. We are grateful to the Austrian Science Fund FWF (Austria) for Grants S-9304-B05, SFB-LIPOTOX and P23490 to FM and DC-G, and to the European Commission for project APOSYS to FM. GK is supported by the Ligue Nationale contre le Cancer (Equipe labellisée), Agence Nationale pour la Recherche (ANR), European Commission (Apo-Sys, ApopTrain, ArtForce, ChemoRes), Fondation pour la Recherche Médicale (FRM), Fondation Bettencourt-Schueller, Institut National du Cancer (INCa) and Cancéropôle lle-de-France.

1. Ding J et al. Appl Microbiol Biotechnol 2009; 85: 253-263.

2. Carmona-Gutierrez D et al. Cell Death Differ 2010; 17: 763-773.

3. Madeo F, Frohlich E, Frohlich KU. J Cell Biol 1997; 139: 729-734.

4. Kitagaki $\mathrm{H}$ et al. FEBS Lett 2007; 581: 2935-2942.

5. Eisenberg T et al. Apoptosis 2007; 12: 1011-1023.

6. Landolfo S et al. Biochim Biophys Acta 2008; 1780: 892-898.

7. Scheffler I. Mitochondria. Wiley-Liss: New York, 1999, pp 246-272.

8. Kitagaki H, Shimoi H. J Biosci Bioeng 2007; 104: 227-230.

9. Green DR, Galluzzi L, Kroemer G. Science 2011; 333: 1109-1112.

10. Wang K, Klionsky DJ. Autophagy 2011; 7: 297-300. 